\title{
Las políticas de atención a la pobreza en México, 1970-2001: de populistas a neoliberales*
}

\author{
Barajas, Gabriela**
}

\section{Resumen}

México tiene una larga historia de políticas y programas gubernamentales de atención a zonas pobres, la cual comienza a finales de la década de los sesenta y llega hasta los días actuales. Sin embargo, y a pesar de su importancia, la historia de tales programas no ha sido escrita. El presente artículo tiene como objetivo comenzar a escribir dicha historia. Para lo cual se propone una periodización que se desprende no sólo de los procesos de rupturas y continuidades entre los programas, sino que se centra en la vinculación que guardan éstos con el modelo de desarrollo económico predomi nante. Se concluye que, para el caso de México, las políticas de atención a zonas o grupos de población específicos en situación de pobreza no aparecen junto con el modelo neoliberal, sino con el agotamiento del modelo anterior, el posrevolucionario. Sería hasta 1997, quince años después cuando comienza a instrumentarse el neoliberalismo en México, que aparece el Programa de Educación, Salud y Alimentación, el primero de su tipo acorde con el modelo neoliberal. A lo largo de la revisión histórica es clara la vinculación de cada uno de estos programas con funciones de control político y legitimación gubernamental, por lo tanto, en este trabajo se definen no como políticas estructurales de "combate" sino coyunturales de "atención" de la pobreza, siempre relacionadas con las cambiantes necesidades de estabilidad política y social de los distintos gobiernos.

Palabras clave: Pobreza, políticas populistas, políticas neoliberales, modelos de desarrollo.

\section{Recibido: 02-03-01 . Aceptado: 02-08-19}

* Agradezco de manera muy especial las opiniones de los dictaminadores anónimos, sin duda enriquecieron el artículo y me sugirieron nuevas líneas de investigación.

** Socióloga, Maestra en Ciencias Sociales por la Facultad Latinoamericana de Ciencias Sociales, sede México. Investigadora Asistente en el Instituto de Investigaciones Dr. José María Luis Mora. Dirección para correspondencia: Cúpulas 122, Colonia Jardines de Sur, Xochimilco, 16050, México D.F. E-mail: GabrielaBarajas@aol.com 


\section{Policies Towards Poverty in Mexico, 1970-2001: From Populist to Neoliberal}

\section{Abstract}

Mexico has a long history of governmental policies and programs for the poor, which began at the end of the decade of the nineteen sixties and continues up until the present. However, in spite of its importance, the history of these programs has not been written. The objective of this article is to begin that history. For this purpose a division by periods is proposed based not only on the processes of rupture and continuity between programs, but also centered on the links between these programs the predominant economic development model. The conclusion is that in the case of Mexico, policies of attention towards specific population zones or groups in poverty situations do not appear as a response to the predominant neo-liberal models, but in response to the failure of previous model, the post-revolutionary model. It was not until 1997, 15 years after the instrumentation of neo-liberalism in Mexico, that the Program for Education, Health and Food, the first of its type in the neo-liberal model, appeared. Throughout this historic revision, the relationship of each of the programs with governmental political control functions becomes clear. Therefore in this paper we define them not as political structures to combat poverty, but as temporary programs of attention to the poor, and related to the always changing needs of political and social stability for each government.

Key words: Poverty, populist policy, neo-liberal policy, development models.

\section{Introducción}

El presente artículo tiene como objetivo revisar el origen, características y evolución de lo que llamamos las políticas de atención de la pobreza en México. Este tipo de políticas en nuestro país surgen a principios de la década de los años setenta y se mantienen hasta la fecha. Tienen como denominador común el que constituyen la expresión de la preocupación gubernamental por los sectores que el discurso oficial ubica como los más desfavorecidos, entre los programas públicos de este tipo destacan: el Programa de Inversiones para el Desarrollo Rural (PIDER, 1970-1982), la Coordinación General del Plan Nacional de Zonas Deprimidas y Grupos Marginados (COPLAMAR, 1976-1983), los Programas de Desarrollo Regional (1983-1988), el Progra- ma Nacional de Solidaridad (PRONASOL, 1988-1995), y el Programa de Educación, Salud y Alimentación (PROGRESA, 1997-2001), éste último, recientemente renombrado como Programa OPORTUNIDADES por el gobierno de Vicente Fox (2000-2006).

Es interesante observar que los primeros programas no surgen con el modelo neoliberal sino como una respuesta a la crisis del patrón de desarrollo anterior, el posrevolucionario, y se mantienen hasta convertirse en el centro mismo del modelo de bienestar social neoliberal. Como destacaremos, tales políticas siempre han estado vinculadas con funciones de control político-social y de legitimación gubernamental. Al privilegiar la estabilidad han dejado en un segundo plano la satisfacción de las demandas sociales, sus resultados no han sido evaluados en 
orden de hacer efectivos los objetivos de bienestar y nivelación social sino en términos de su capacidad de desactivar focos de tensión política o social. De esta manera, su permanencia no se explica por el éxito en la reducción de los niveles de pobreza en el país. Como podemos observar en el Cuadro 1, a pesar de que se han instrumentado políticas anti-pobreza durante las últimas tres décadas, y en situaciones económica cambiantes (importantes préstamos del exterior 1970-1976; auge petrolero 1976-1982, políticas neoliberales a partir de 1982, y crisis económica en 1982 y 1994), la incidencia del problema en México ha sido creciente. Todo lo anterior nos lleva a hacer referencia a lo largo del texto a políticas o programas de "atención" y no de "combate" o "lucha contra" la pobreza.

Al revisar esos treinta años de las políticas de atención a la pobreza en México (1970-2001), identificamos tres etapas: una primera que abarca de 1970 a 1982 e incluye como principales programas al PIDER y COPLAMAR. Como analizaremos más adelante, este tipo de programas surgen a principios de la década de los setenta cuando comenzaron a hacerse patentes, de forma sistemática, las crecientes dificultadas económicas a las que se enfrentaba el Estado posrevolucionario, y la cada vez más evidente debilidad de los mecanismos tradicionales de control político corporativo. Es claro que estaban diseñados para moldear y dar cauce institucional a las demandas de grupos de población que se encontraban al margen de las entonces poderosas estructuras corporativas. En esta primera etapa, los mencionados programas no implicaron una redefinición del papel del Estado respecto de su compromiso con el bienestar de los sectores populares, al contrario, es importante ubicarlos como parte de los esfuerzos gubernamentales por darle un nuevo sentido al proyecto estatista posrevolucionario. No constituyeron una política específica de combate a la pobreza, sino una política de desarrollo nacional aplicada a zonas definidas como particularmente pobres.

Durante la segunda etapa, ubicada de 1982 a 1994, se instrumentó el PRO. NASOL salinista (1988-1995); ésta la definimos como una etapa de transición. Teniendo como marco el cambio en el modelo de desarrollo y el repliegue del Estado de sus responsabilidades con el bienestar social general, el PRONASOL es presentado como el primer programa de combate a la pobreza en nuestro país. Sin representar cambios tan profundos a nivel operativo, programático ni de presupuesto asignado como lo planteó el discurso gubernamental, su importancia se proyectó en términos ideológicos: sirvió como fórmula de gobernabilidad y permi-

\section{Cuadro 1 \\ Evolución de la pobreza en México \\ (\% de Hogares)}

\begin{tabular}{|l|l|l|l|l|l|l|}
\hline 1968 & 1977 & 1984 & 1989 & 1992 & 1994 & 1996 \\
\hline $34 \%$ & $32 \%$ & $34 \%$ & $39 \%$ & $36 \%$ & $36 \%$ & $43 \%$ \\
\hline
\end{tabular}

Fuente: (Boltvinik, 1999:19). 
tió redefinir el pacto social posrevolucionario; a partir de ese momento, el Estado solo estaría comprometido con el bienestar de los pobres extremos y ya no con las clases populares.

La tercera etapa abarca de 1995 al gobierno actual, de Vicente Fox. Sentadas las bases del modelo neoliberal, en esta etapa se está configurando un patrón de política social propio de tal modelo, cuyo elemento central es el PROGRESA, hoy OPORTUNIDADES. Dicho programa, en un contexto de eliminación de subsidios generalizados, ya no focaliza a su población objetivo a partir de las demandas de los beneficiarios potenciales, como se hizo desde el PIDER hasta PRONASOL, sino a través de un padrón de familias que se encuentran por debajo de la línea de la pobreza en el sector rural. Prácticamente, ya no se apoyan proyectos productivos, y por primera vez se diseña un programa compensatorio. Un modelo de acuerdo con la lógica de mercado y la exigencia de eficiencia técnica en la atención de la pobreza. Comencemos nuestra revisión.

\section{Las Políticas Populistas de Atención a la Pobreza: el PIDER y la COPLAMAR}

La primera etapa de nuestra revisión histórica abarca de 1970 a 1982, e incluye los sexenios de Luis Echeverría Álvarez y José López Portillo. A lo largo del apartado destacamos el surgimiento del primer programa de atención a la pobreza en nuestro país, y ubicamos como éste y otros programas similares son parte de los esfuerzos gubernamentales por reactivar el modelo posrevolucionario de desarrollo.

\subsection{EI PIDER}

Durante el gobierno de Luis Echeverría Álvarez (1970-1976) aparece el primer programa de atención de la pobreza en nuestro país: el PIDER. Dentro de un contexto de estancamiento económico y potencial inestabilidad política y social $^{1}$, el Presidente Echeverría reconoció al momento de su toma de posesión: "subsisten graves carencias e injusticias

1 Desde finales de la década de los sesenta, entre los investigadores sociales se había generado un especie de consenso respecto al carácter estructural de los problemas que enfrentaba el Estado. A nivel económico, identificaron una serie de cuellos de botella y señalaron la necesidad de redefinir el modelo de desarrollo hasta ese momento promovido (Medina, 1994:169-176). En el nivel político también existía preocupación. La matanza de estudiantes por parte del ejército en 1968 mostró con nitidez los riesgos de una estructura estatal cada vez más cerrada e impermeable a las demandas y aspiraciones de diferentes capas de la sociedad (Woldenberg, 1986:160). Además de que tales hechos fueron vistos como preludio de un conflicto social mayor (Trejo y Jones, 1993:174). Por último, la existencia de la guerrilla urbana y rural era evidencia clara de que el México mítico de "la estabilidad política, la paz, y el incesante progreso, el orden y la tranquilidad social", estaba en entredicho. 
que pueden poner en peligro nuestras conquistas: la excesiva concentración del ingreso y la marginación de grandes grupos humanos amenazan la continuidad económica del desarrollo" (Woldenberg, 1986: 159).

Tres hechos nos permiten entender el surgimiento de la preocupación gubernamental por el problema de la pobreza. Primero, el reconocimiento no sólo de que el crecimiento económico por sí mismo no garantizaba una mejor distribución del ingreso sino también que la riqueza de unos pocos había sido a costa de la mayor pauperización de las masas populares. Así lo anota un diagnóstico oficial sobre la situación del campo: "En el medio rural se observa una concentración del ingreso y la riqueza en los productores agrícolas ubicados en las zonas de riesgo (...); los intermediarios (...); los ganaderos particulares, (...); y los concesionarios privados de bosques con talas irracionales. Frente a éstos, se encuentra la mayor parte de la población del medio rural que habitaba en zonas de temporal con pésimas condiciones de vida en materia de nutrición, salud, educación y vivienda, que por falta de apoyo empiezan a dejar de producir los alimentos necesarios para su propia subsistencia y emigran a las zonas urbanas" (SPP, 1985: 197. Cursivas nuestras).

Segundo hecho importante a señalar, es que a nivel internacional, los organismos financieros planteaban la reducción de la pobreza como una condición primordial para lograr el desarrollo. El Banco Mundial anotaba que se requerían programas para elevar el nivel de vida y la capacidad productiva de la población campesina. Aspecto que señaló el propio director del Banco Mundial en 1973: "A menos que se tomen medidas para beneficiar directamente a los segmentos más pobres de la población, las desigualdades del ingreso sencillamente se intensificarán. Creo, por consiguiente, que si se quiere lograr un progreso significativo hacia la solución del problema de la pobreza absoluta en las zonas rurales, no existe otra alternativa viable que la incrementar la productividad de la pequeña agricultura" (McNamara, citado por: Valencia y Aguirre, 1998:45).

Por último, la crisis agrícola de principios de las década de los setenta; la cual afectó de manera más severa a los productores de zonas de temporal, los más pobres. La creciente pobreza de la población rural, sumada a la crisis de las centrales campesinas (sobre todo de la Confederación Nacional Campesina, organización del Partido Revolucionario Institucional, la cual mostraba una creciente incapacidad para promover la satisfacción de las demandas de tierra), explican el surgimiento de un desorganizado pero creciente movimiento campesino, cuyas armas fueron la toma de tierras, las marchas a la ciudad de México, las tomas de locales de organismos oficiales agrarios y la organización de frentes y agrupaciones locales y regionales independientes del Estado (Castell y Rello, 1981:619-621). Estas movilizaciones no tenían precedentes en la historia del país; además, sus demandas eran múltiples: presiones por mejores precios para sus productos, luchas contra los caciques, huelgas de trabajadores agrícolas, y sobre todo la pelea por la tierra (Montes de Oca, 1981:597).

La propuesta gubernamental para la solución a la problemática rural estuvo 
acompañada de una mayor intervención del Estado en el sector. Se intentó modernizar a la agricultura tradicional con inyección de capital, y propiciar la colectivización de ejidos que tuvieran tierras de riego y con potencial comercial. La inversión en el campo fue muy alta, creció a un ritmo de 21 por ciento anual, se pasó de 12 por ciento del total de la inversión pública en 1970, al 18 por ciento en 1975 (Campos, 1995: 229-230). De manera específica para la atención de las llamadas zonas rurales atrasadas se crearon una serie de fideicomisos y se ampliaron las acciones de dependencias ya existentes. Por ejemplo, se creó el Programa de Obras Rurales, el de Unidades de Riego para el Desarrollo Rural; además de una serie de nuevos organismos como la Comisión Nacional de Zonas Aridas (1970), el Patrimonio Indígena del Valle del Mezquital (1972), Productores Forestales de la Tarahumara (1972) y el Fideicomiso para Obras Sociales a Campesinos Cañeros de Escasos Recursos (s/f), entre otros. Con el fin de apoyar el desarrollo de las zonas rurales pobres, se ampliaron las acciones del Instituto Mexicano del Seguro Social (IMSS) e incrementaron de manera importante las actividades de la Compañía Nacional de Subsistencias Populares (CONASUPO) para atender tanto los mercados rurales y urbanos. Por último, se agrandó la estructura administrativa del Instituto Nacional Indigenista, el cual pasó de contar con 13 Centros Coordinadores Indigenistas en 1970 a 64 en 1976. Además, de que su presupuesto creció, en términos absolutos, poco más de 700 por ciento: aumentó de 27327932 pesos en 1970, a 220100000 pesos en 1976 (INI, 1977:503). Sin embargo, como anota un diagnóstico oficial, los esfuerzos terminaron siendo "aislados con ninguna o muy poca coordinación entre unos y otros" (SPP, 1985:256).

Dentro del anterior marco político, social y administrativo, surgió en 1973 , el Programa de Inversiones para el Desarrollo Rural (PIDER) como un proyecto de desarrollo rural financiado por el Banco Internacional de Reconstrucción y Fomento (BIRF) y del Banco Interamericano de Desarrollo (BID) ${ }^{2}$. Con el Programa se coordinó recursos en 200 zonas "atrasadas", "para proporcionar infraestructura física y económica; promover producción agrícola, pecuaria, minera, pesquera e industrial y, a la vez, servicios de salud y vivienda, sin faltar la asesoría técnica y organizativas". (Campos, 1995:231). En el discurso, PIDER no se presentaba como una política específica de atención a la pobreza, se insertaba dentro de una política nacional de desarrollo aplicada, en este caso, a comunidades rurales con carencias extremas. Aquí, lo que vale la pena destacar es que representó una nueva forma de atender las demandas de grupos sociales específicos, además de

2 En 1973, México obtuvo del BIRF un primer crédito por un monto de 110 millones de dólares para ser aplicado a 30 microrregiones en 5 años. La novedad del enfoque del PIDER hizo que el BID concediera un préstamo de 20 millones de dólares en 1976 para ser utilizado en 15 microrregiones. Ambos créditos fueron aplicados en su totalidad (SPP, 1985:201). 
que en términos administrativos resultó novedoso.

Respecto a esta última cuestión, el PIDER rompió con un procedimiento tradicional dentro del aparato público mexicano (particularmente del segmento responsable del desarrollo agropecuario), que era el promover la creación de organismos o dependencias ejecutoras para cualquier necesidad específica, lo cual llevaba a la duplicidad de funciones. Por el contrario, el Programa buscó coordinar las acciones de las grandes Secretarías de Estado en programas y proyectos específicos de desarrollo para regiones y comunidades rurales "atrasadas", asignándoles a las Secretarías recursos financieros adicionales a los de sus presupuestos normales (SPP, 1985:201). El PIDER fue el primer mecanismo administrativo en la historia de México que basó su operación en una coordinación y cooperación de las dependencias federales a nivel nacional y estatales ${ }^{3}$.

Se buscaban mayores niveles de eficiencia administrativa y política, a manera de respuesta frente a un aparato administrativo que no respondía a las necesidades sociales de los grupos de población con poca capacidad de articular sus demandas y presionar para que fueran escuchadas. Lo que hacía el Programa era ubicar las necesidades sociales y económicas de los sectores de la pobla- ción pobres antes de que partiera de ellos las demandas convertidas en protesta social, lograr lo anterior generaba reconocimiento popular. Por otro lado, dentro del aparato administrativo, el PIDER se convirtió en un flexible mecanismo de control sobre diferentes Secretarías de Estado en manos del titular del Poder Ejecutivo, lo anterior desde el momento en que la asignación de los recursos del PIDER se realizaba desde la Presidencia. De esta forma, el PIDER, y programas posteriores similares, funcionaron tanto como un vínculo entre el Presidente (que siempre encabezaba tales iniciativas) y los grupos de pobres beneficiarios de las acciones realizadas, así como un mecanismo presidencial de control sobre importantes Secretarías.

Para finalizar, cabe destacar que dentro de los objetivos del PIDER se le dio una singular importancia al tema de los apoyos a los proyectos productivos. No obstante, se le asignó solo el $38 \%$ de los recursos manejados por el Programa entre 1973 y 1976 (SPP, 1985:198). El menor peso relativo de las inversiones destinadas a promover directamente la producción, se justificó por la necesidad de cubrir la escasa infraestructura como caminos, electrificación, aulas, centros de salud y agua potable, ante el abandono en que se encontraban las áreas rurales del país (SPP, 1985:201).

3 Para lograr la participación de las dependencias federales en el PIDER, se creó un Comité Coordinador presidido por el Director de Inversiones Públicas, dependiente de la Secretaría de la Presidencia; se establecieron comités estatales, coordinados por un vocal ejecutivo del gobierno del estado, al interior del cual participaban los representantes de las dependencias federales en la entidad (SPP, 1985:200). 
Es interesante destacar que la anterior práctica político-administrativa se convirtió en una característica de los programas de atención de la pobreza hasta mediados de la década de los noventa. Característica que podemos explicar por el hecho de que a la administración pública le resultaba más fácil instrumentar acciones de infraestructura social básica (electrificación, alcantarillado, agua potable, pavimentación, etc.), que proyectos productivos; los cuales implicaban, muchas veces, confrontarse con los intereses de los cacicazgos locales que por lo regular controlaban los canales de comercialización y venta de productos agropecuarios (Szekely, 1993:3-4). Promover directamente la producción resultaba administrativa y políticamente más complicado, por lo tanto, el impulsar pequeñas obras de servicios básicos permitía satisfacer un mayor número de demandas. Evidentemente, lo que importaba era la legitimidad que la gestión del Presidente Echeverría obtenía a través de tales acciones. Cabe señalar que al final del sexenio no hubo evaluación del impacto de las acciones del PIDER.

\subsection{La COPLAMAR}

Cuando José López Portillo asumió la Presidencia de la República (19761982), el país pasaba por otra profunda crisis económica y política. Crisis que perfilaba cada vez más claro, el carácter estructural de las dificultades económicas y políticas a las que se enfrentaba el Estado posrevolucionario.

El Presidente buscó resolver las enormes dificultades políticas y económicas, de nueva cuenta, fortaleciendo la ca- pacidad rectora del Estado, ahora a través de una importante Reforma Administrativa. La situación por la que atravesaba el aparato administrativo mexicano era difícil. El crecimiento desmesurado de la administración pública durante el gobierno de Echeverría agravó los conflictos por la superposición de acciones, la duplicidad de funciones y redundancia de competencias. Por ejemplo, el Programa de Desconcentración Administrativa echeverrista acentuó el problema de la duplicación de funciones al ensanchar la esfera de competencias federales. Este programa llevó a que la Secretaría de Hacienda y Crédito Público, la Secretaría de Industria y Comercio, y la Secretaría de Obras Públicas, establecieran oficinas al interior del país; otras, como las Secretarías de Agricultura y Ganadería, y la Secretaría de Recursos Hidráulicos, fortalecieron sus representaciones estatales, y fueron mínimas las facultades de decisión delegadas, al contrario, se disminuyó la esfera de competencia local (Ortega, 1988:134). Dentro de este contexto, la administración de López Portillo consideró la cuestión de la pobreza como un problema resoluble mediante la reorganización, ya no crecimiento, del aparato administrativo responsable de su atención.

La estrategia antipobreza lopezportillista supuso similitudes importantes con respecto al gobierno de Luis Echeverría. Primero, de nueva cuenta se reconoce la vinculación del tema de la estabilidad política y social con los problemas de la desigualdad y la pobreza en la que vivían importantes sectores de la población. Como se anota en un documento oficial: "La brecha de la desigualdad social se ha ensanchado peligrosamente y 
que de no multiplicar en forma coherente una serie de acciones en favor de los pobres, las actuales circunstancias y su tendencia, multiplicarán, en cambio, los conflictos sociales y pueden llegar a afectar peligrosamente la estabilidad política y la paz social del país" (COPLAMAR, 1977A:5).

Segundo, las políticas de atención de la pobreza se concentraron en el sector rural. El gobierno lopezportillista desarrolló tres líneas de acción: se creó el Sistema Alimentario Mexicano ${ }^{4}$; continuaron las acciones del PIDER, y, por último, se estableció la COPLAMAR. Ésta asume, por primera vez, el combate a la marginación como objetivo explícito.

Tercero, la COPLAMAR, lo mismo que el PIDER, no fue un programa sino un sistema de coordinación administrativa. Al ser presentado en julio de 1977, el propio Presidente lo definió: "Este es simplemente un sistema de optimización de recursos establecidos e identificados por su destino específico: servir a los marginados, al que habrán de concurrir todas las entidades, prácticamente todas tienen acción en esta área" (COPLAMAR, 1977A:39).

Por último, al igual que el sexenio anterior, las acciones de atención de la pobreza no suponían una política específica. Para el gobierno de López Portillo la política nacional y la de marginados "es una y la misma, sólo que el Ejecutivo Federal desea dar a este segundo aspecto un énfasis singular" (COPLAMAR, 1977A:17).
En enero de 1977, se crea por decreto presidencial la COPLAMAR que estaría integrada por las entidades públicas especializadas para atender los problemas derivados de la marginalidad social y el atraso económico (Instituto Nacional Indigenista, Comisión Nacional de Zonas Aridas, Patrimonio Indígena del Valle del Mezquital, La Forestal FCI, Fondo Candilillero, Productores Forestales de la Tarahumara, Patronato del Maguey, Fondo Nacional para el Fomento de las Artesanías, Compañía Forestal de la Lacandona y Fideicomiso de Desastres). Se trataba, así lo destaca el presidente López Portillo, de "evitar la dispersión de la acción pública en las zonas marginadas y establecer esquemas de seguimiento, en campo, de los programas y acciones interinstitucionales basados en la participación de las comunidades y el aprovechamiento de la penetración territorial de las entidades agrupadas en la Coordinación" (COPLAMAR, 1997B: 39).

Efectivamente, la razón de ser de la COPLAMAR era la idea de aprovechar la penetración territorial de las dependencias integrantes. Las regiones y áreas donde trabajaban las citadas dependencias fueron definidas como las prioritarias; de esta manera se ubicó una población objetivo compuesta por 18 millones de habitantes entre indígenas y campesinos pobres del desierto, del semidesierto y de las zonas montañosas de México. Eran importantes los recursos que poterno y elevar los ingresos y la productividad del campesino pobre. Al respecto consultar (Arteaga, 1985). 
dían ofrecer las entidades agrupadas en COPLAMAR: 140 oficinas que podían desempeñar la función de unidades de vinculación programática con las zonas de trabajo y 4,700 empleados (COPLAMAR, 1977A:29-30). Era claro el objetivo, lograr que todo el andamiaje humano y material mencionado articulara "las diversas demandas de la población y las canalicen de manera integrada, jerarquizada y complementaria hacia las dependencias y entidades de la administración competentes" (COPLAMAR, 1997A:28).

De esta manera, tanto PIDER como COPLAMAR buscaron conformarse como sistemas de coordinación administrativos cuyo fin era detectar y encauzar institucionalmente las demandas sociales. Documentos oficiales de ambos programas reconocieron que la solución al problema de la pobreza se relacionaba con el funcionamiento del conjunto del sistema económico, entonces, lo que buscaban estas políticas era ubicar las demandas de los sectores rurales pobres que habían estado al margen de la acción del aparato público. Solucionar las necesidades era menos importante que detectarlas y encauzarlas administrativamente antes de que se convirtieran en inestabilidad social.

La puesta en marcha de la COPLAMAR no fue un proceso sencillo. El cargo de Coordinador General fue asignado a Ignacio Ovalle Fernández, al mismo tiem- po, director general del Instituto Nacional Indigenista (INI).

La designación de Ovalle no era casual, reflejó el papel central a jugar por el INI en el diseño e instrumentación de la COPLAMAR. Creado en 1948, el INI recibió un apoyo sin precedente durante el sexenio de Echeverría. Durante el gobierno de López Portillo, el INI tenía los recursos humanos y organizativos para promover obras y servicios en muchas de las zonas rurales marginadas del país. En muchos casos, los Consejos Coordinadores Indigenistas fueron los núcleos de la actividad central de programación en las regiones. "La única institución que sabía qué localidad tenía caminos de herradura y cuántos habitantes, era el Instituto. (...) El Sistema [COPLAMAR] arranca con la cobertura del Instituto, con los Centros Coordinadores Indigenistas. Te puedo decir que el 80 por ciento de todos los Almacenes [CONASUPO] están donde eran sedes del Instituto Nacional Indigenista" (Entrevista $A)^{5}$.

Lo que hacía particular al INI era que: "la presencia del Instituto era permanente, asambleísmo, reuniones de trabajo, reuniones de evaluación". (Entrevista B) 6 "Mucha gente del 68 [participantes del movimiento estudiantil] se empezó a ubicar en ciertos programas, de CONASU. PO-COPLAMAR, de empleo temporal; y esa gente traía ya otra visión del trabajo social, esto permitió que COPLAMAR,

5 Entrevista de la autora con un funcionario del programa CONASUPO-COPLAMAR, quien laboró en tal programa de 1979 a 1982; en adelante (Entrevista A).

6 Entrevista de la autora con un funcionario del INI, quien laboró en el Instituto de 1973 a 1983; en adelante (Entrevista B). 
pudiera retomar, yo creo, un impulso importante en el campo" (Entrevista B).

De esa manera, el perfil de los funcionarios del Instituto era muy particular. Eran personas: "que tenían arraigo con las comunidades, tenían el conocimiento de la problemática, tenían el conocimiento físico de la región, tenían el conocimiento de las estructuras de poder, tenían todo un conjunto de elementos que hacen ser el personal idóneo para realizar ese tipo de acciones en campo" (Entrevista A).

Además de contribuir con sus recursos humanos y la cobertura territorial, otra aportación importante del INI fue el énfasis dado a la participación y organización comunitaria. Inspirados en prácticas como el tequio, mayordomía, chivitú, convite, guelaguetza, gazona, techa y mano vuelta (costumbres propias de los grupos indígenas del país), en los programas de COPLAMAR se promovieron la formación de comités de beneficiarios y la obligación de las localidades de aportar trabajo comunitario. Lo anterior queda muy claro con programas como CONA. SUPO-COPLAMAR (CONASUPO, 1982: 14-19) e IMSS-COPLAMAR (IMSS-COPLAMAR, s/f:2).

Contar con funcionarios de amplia experiencia en trabajo de base y aprovechar la forma de organización de las propias comunidades al instrumentar las políticas de atención de la pobreza, fueron dos de las aportaciones centrales del COPLAMAR a programas posteriores.

A pesar del total apoyo otorgado por el Presidente, los resultados fueron poco alentadores. Durante 1980, luego de que "se revisó en la presidencia el avance del programa y se supo que en estos primeros tres años no se había hecho nada" (Campos, 1995:188). Fue entonces que se asignaron directamente recursos financieros a COPLAMAR, como se había hecho con el PIDER el anterior sexenio. Los recursos se concentraron en un ramo presupuestal, el Ramo XXVI denominado "Promoción Regional", del cual se asignaban a las dependencias ejecutoras para después aplicarlos en las zonas definidas por el INI como marginadas. Los recursos eran intransferibles.

Ante la negativa de las Secretarías de colaborar en el Sistema COPLAMAR, el Poder Ejecutivo volvió a utilizar el conocido mecanismo para obligarlas a atender las zonas marginadas: el manejo desde Presidencia de recursos extras a los presupuestos normales asignados a cada Secretaría. Lo mismo que con el PIDER de Echeverría, no hubo una evaluación de los resultados de las acciones y obras promovidos por COPLAMAR. Sin embargo, de manera general podemos anotar que la pretensión de atender simultánea y rápidamente diversos aspectos del problema de la pobreza en estas comunidades, llevó "según diversos estudios del tema, a la duplicidad de funciones y al dispendio" (Valencia y Aguirre, 1998:59); aquello, precisamente, que según el discurso presidencial de López Portillo se trataba de evitar.

A finales del sexenio lopezportillista sucedió la caída de los precios del petróleo y el alza de las tasas de interés internacionales que pusieron fin al boom económico interno. La dimensión de la crisis económica mostró que los cambios promovidos a lo largo de la década de los setenta no habían sido suficientemente profundos para reactivar el patrón de acu- 
mulación de capital basado en el proyecto industrializador proteccionista. El surgimiento de un nuevo patrón de desarrollo económico-político (el neoliberal), no sería fácil y supondría rupturas y continuidades con el anterior modelo. Entre las continuidades estuvieron las políticas de atención de la pobreza, las cuales se convertirían en esenciales para el nuevo patrón de desarrollo.

\section{Entre la Política Populista y Neoliberal de Atención a la Pobreza: los Programas de Desarrollo Regional y el PRONASOL}

La segunda etapa de nuestra revisión abarca de 1982 a 1994, e incluye los sexenios de Miguel de la Madrid (19821988) y Carlos Salinas de Gortari (19881994). La consideramos de transición porque durante estos gobiernos, particularmente en el segundo, se retomó la forma tradicional de funcionamiento de $\mathrm{PI}$ DER y COPLAMAR (operar a partir de las demandas acotadas de los grupos organizados definidos como pobres), para impulsar o sentar las bases del nuevo modelo de desarrollo: el neoliberal.

\subsection{Los Programas de Desarrollo Regional}

Al inicio del gobierno de Miguel de la Madrid, el país se encontraba en una honda crisis económica y política. En este contexto comenzó el desmantelamiento del proyecto estatista, el cual sufría un enorme desprestigio luego de una década de esfuerzos fallidos por renovarlo.

Durante 1983, la crisis de la deuda obligó a Miguel de la Madrid a firmar una carta de intención con el Fondo Monetario Internacional en la cual se comprometía a promover un profundo programa de estabilización y ajuste. Tal programa supuso el achicamiento del Estado (venta, fusión y/o liquidación de un número grande de empresas públicas), y la reducción del gasto público ${ }^{7}$, la cual se tradujo en la suspensión de una serie de subsidios, cancelación de programas sociales y despido de empleados públicos. Todo ello llevó a la redefinición del papel del Estado en la economía, la suya sería una labor de regulación, control y orientación ya no una participación directa; y en materia de bienestar social se buscaría limitar su intervención al mínimo. Así comenzó la definición del modelo de desarrollo

7 Entre 1983 y 1988, el presupuesto federal no creció en términos reales, además tuvo una distorsión financiera creciente. En 1981, el $70 \%$ del gasto se destinaba a los renglones productivos y sociales, el $24 \%$ al servicio de la deuda y el resto representaba participaciones a estados y municipios; al año siguiente la proporción entre gasto programable y servicio de la deuda fue de $39 \%$. Seis años después esta distorsión llegó a invertir las proporciones: en el presupuesto para 1988 sólo el $38 \%$ se destina a bienes y servicios y, por el contrario, el $57 \%$ tiene como objeto el pago del servicio de la deuda pública interna y externa (Carrasco y Provencio, 1988:92). 
económico y de intervención del Estado neoliberal.

En relación con el problema de la pobreza, es común pensar que durante el gobierno de Miguel de la Madrid nada se hizo ya que tal tema fue eliminado de la retórica oficial. Sin embargo, vale la pena destacar dos hechos. Primero, COPLAMAR si bien desapareció (en abril de 1983 se derogó el acuerdo de su creación), se mantuvieron la serie de programas sectoriales realizados a través de dicha Coordinadora. Los programas quedaron bajo la responsabilidad de las dependencias federales encargadas de instrumentarlos. Por ejemplo, el Programa de Solidaridad Social por Cooperación Comunitaria pasó a ser responsabilidad del Instituto Mexicano del Seguro Social; el Programa de Electrificación para Zonas Marginadas del Medio Rural a la Comisión Federal de Electricidad; el Sistema CONASUPO-COPLAMAR de zonas marginadas y servicios integrados de apoyo a la economía campesina a la CO NASUPO; el Programa de Agua Potable y Caminos en lo relativo a caminos a la Secretaría de Comunicaciones y Transportes; el Programa para el Establecimiento de Casas Escuela a la Secretaría de Educación Pública; y el Programa de Capacitación y Empleo Cooperativo para el Fomento de Recursos Naturales a la Secretaría de la Reforma Agraria (Valencia y Aguirre, 1998:62).

Segundo, fue a lo largo del gobierno de Miguel de la Madrid cuando se sentaron las bases operativas para la instrumentación de programas de atención a la pobreza posteriores, nos referimos a los Convenios Únicos de Desarrollo
(CUD’s). Durante el gobierno de López Portillo, los Convenios Únicos de Coordinación, antecedentes de los CUD's, fueron las herramientas para planear inversiones conjuntas entre la Federación y los estados, financiadas a través de un ramo específico del presupuesto de egresos federal, el Ramo XXVI denominado "Promoción Regional". Con Miguel de la Madrid, de manera inicial, mantuvieron la misma estructura con distinto nombre: Convenios Únicos de Desarrollo, y el Ramo XXVI se llamó de "Desarrollo Regional". Estaban integrados por los siguientes programas: Sectoriales Concertados (PROSEC); de Desarrollo Estatal (PRODES); Programas Estatales de Inversión (PEI); Integral para el Desarrollo Rural (PIDER) y de Atención a Zonas Marginadas (PAZM), éste último concentraba los recursos ejercidos antes por la COPLAMAR. En 1984 cambiaron, y se acordó unificar los cinco programas que integraban los CUD's (PEI, PIDER, PAZM, PROSEC, PRODES) bajo el rubro de "Programas de Desarrollo Regional", con tres especialidades programáticas: a) Programas de infraestructura básica de apoyo), b) Programas de infraestructura para el desarrollo social; y c) Programas productivos. "Con esta unificación de programas se llevó a cabo un proceso único de programación con una sola apertura programática, una propuesta de inversión de los gobiernos estatales, un mecanismo único de administración de recursos y la agilización en los movimientos presupuestales" (SPP, 1985: 363). Con lo anterior estaban dadas las bases administrativas para instrumentar el Programa Nacional de Solidaridad (PRONA- 
SOL), el cual hasta la fecha ha sido el programa de atención a la pobreza más ambicioso en la historia del país.

\subsection{El Programa Nacional de Solidaridad}

El inicio del sexenio de Carlos Salinas de Gortari resultó ser particularmente conflictivo a nivel político. Durante la campaña electoral presidencial de 1988, fue evidente el descontento de amplias capas de la población por el deterioro de su nivel de vida provocado por el programa de ajuste económico que impuso el gobierno de Miguel de la Madrid ${ }^{8}$. El descontento cristalizó en un amplio movimiento social poselectoral que objetaba violentamente una de las reglas no escritas del sistema político mexicano: el fraude electoral.

Los beneficios iniciales de la reforma estructural delamadrista se había concentrado fundamentalmente en el sector exportador, desatendiendo a la mayoría de la población. De esa manera, para el gobierno de Salinas, la gran lección del rechazo electoral fue que no se podía instrumentar un programa de cambio político y económico vertical sin el apoyo de grupos sociales organizados (ver: Dresser, 1997). El modelo de desarrollo neoliberal, encabezado por un Estado adelgazado que promovería el creci- miento económico mediante el apoyo principalmente al sector privado exportador, requería de forma urgente de una nueva alianza que sirviera de base social.

Sin embargo, los retos que enfrentaba el gobierno de Carlos Salinas no solo eran políticos, el panorama económico no era menos problemático. Para diciembre de 1988, la economía nacional presentaba síntomas de estancamiento prolongado y altas tasas de inflación. La causa principal de la situación era la enorme transferencia de recursos al exterior por concepto de la deuda, sencillamente, tal salida de capital era incompatible con la reactivación económica. Entre 1983 y 1988, la salida de recursos significó el 6 por ciento del PIB, y para 1988 la deuda ascendía a más de 100 mil millones de dólares, es decir, el 57 por ciento de los recursos generados por el país ese año (Medina, 1994:250). Pero un año le bastó a Salinas para recuperar el control del país, pasar a la ofensiva y comenzar a impulsar el proyecto de modernización que parecía estancado.

La serie de reformas instrumentadas incluyeron tres reformas político-electorales, el desmantelamiento del intervencionismo estatal (adelgazamiento del Estado, liberalización comercial y reducción de la regulación del mercado), una nueva reforma administrativa (Aguilar, 1996:198), y, en materia de política so-

Para 1988, el desempleo había alcanzado la cifra de 7 millones de desocupados, el 20 por ciento de la PEA. A lo largo del sexenio, el salario mínimo sufrió una pérdida del 52 por ciento (Gutiérrez, 1989:14). El gasto público para el bienestar pasó de 17.2 por ciento del presupuesto total en 1981 a 9.2 de ese total en 1987. El gasto de bienestar se redujo de manera proporcional al aumento de los recursos destinados al pago de la deuda (Gutiérrez, 1989:101). 
cial, la promoción de programas focalizados. Dentro de este contexto surge el PRONASOL.

Este programa conocido también como Solidaridad, lejos de ser un programa de emergencia social como los promovidos en esos momentos por el Banco Mundial $^{9}$, fue muy cercano a las experiencias de políticas de atención de la pobreza como PIDER y COPLAMAR.

El Programa fue establecido por acuerdo presidencial el 2 de diciembre de 1988, y representó el primer acto de gobierno del Presidente Salinas, lo cual resulta muy importante dada la difícil coyuntura en la que ascendió al poder. Luego de estar ausente de la retórica oficial durante un sexenio, el tema de la pobreza pasó a ser el centro mismo del discurso del Presidente Salinas. Al igual que administraciones anteriores, la preocupación por la pobreza estuvo cruzada por el tema de la estabilidad política y social. El diagnóstico era claro: "hay que hacer un gran esfuerzo para intentar igualar capacidades, pero sobre todo hay que evitar que la estratificación llegue a obstaculizar el libre juego de las oportunidades y le imprima al sistema una rigidez explosiva" (Consejo Consultivo, 1991:60).

Sobre el funcionamiento de Solidaridad cabe destacar la rapidez con que fue instrumentado, y su capacidad para responder a las demandas sociales. Lo anterior se explica porque PRONASOL se montó sobre instrumentos programáticos y administrativos heredados de los sexenios anteriores (Ramo XXVI "Desarrollo Regional" y los Convenios Únicos de Desarrollo), además de que recuperó también a ese "equipo de operadores familiarizados con el trabajo y el contacto directo con las comunidades" (SEDESOL, 1993:42), cuya capacidad de penetración territorial era conocida desde la época de la COPLAMAR.

Al inicio del gobierno, Carlos Salinas sencillamente continuó con lo realizado a lo largo de la administración de Miguel de la Madrid. Sin embargo, ese año (1989) se realizaron dos cambios en el funcionamiento de los Convenios Únicos de Desarrollo que implicaron una mayor centralización del Programa en torno de la figura presidencial.

1) Por primera vez, los sectores de la administración pública federal se comprometieron a que todos los recursos financieros que se transfirieran a los gobiernos de los estados se harían única y exclusivamente por el Ramo XXVI "Desarrollo Regional" (Vázquez, 1989:254). La anterior medida se justificó como una acción que buscaba una mayor racionalidad del aparato público, pero en los hecho significó la concentración de los recursos

Llamados también Fondos Sociales, dichos programas estaban sustentados bajo criterios de "eficiencia técnica" y buscaban lo que llamaron "compensación social", focalizando "poblaciones objetivo" (los grupos más pobres), con la idea de darles una "red de protección" mediante paliativos de ingreso, alimentación o empleo por el tiempo que durara el ajuste económico. Además de tener una temporalidad definida (por lo general, cuatro años), utilizaban estructuras extraestatales para su aplicación (organizaciones no gubernamentales) y eran financiados con recursos predominantemente externos. Al respecto ver (Barajas, 1997). 
en manos del Presidente quien los manejaría con la enorme discrecionalidad que permitía el Ramo XXVI. Lo anterior llevó a un proceso de centralización alrededor de Solidaridad de todos los programas realizados por la administración federal en los estados, especialmente aquellos que llevaban a cabo trabajo de base o apoyo comunitario. De los trece programas que inicialmente conformaron PRONASOL "once existían con anterioridad, con nombres, propósitos, diseño y operación muy semejantes" (Peniche, 1992: 22). Lo anterior explica el crecimiento espectacular de Solidaridad. Por ejemplo, la incorporación de programas como el de Integración de la Mujer al Desarrollo (PINMUDE) o de IMSS-COPLAMAR, presentados en 1989 como Mujeres en Solidaridad e IMSS-Solidaridad respectivamente, aportaron métodos de trabajo comunitario, oficinas en campo, personal especializado en trabajo de base y una importante estructura organizativa a nivel comunitario, producto de la labor realizada en miles de poblados a lo largo de más de una década a través de programas como PIDER, COPLAMAR y otros.

2) La incorporación al Convenios Únicos de Desarrollo, por primera vez, de un anexo técnico que describía detalladamente las acciones convenidas, mismo que se agregaba al Convenio al momento mismo de su firma (Vázquez, 1989:255). Dicho cambio fue justificado por la necesidad de dar a conocer a los gobiernos estatales la ubicación, monto y responsables de la ejecución de las acciones y obras coordinadas. En la práctica, los anexos contenían el detalle de obra por obra y lugar por lugar donde se ejecutaría cada acción de beneficio social, pero además se exigía que cada una de ellas fuera avalada por la organización social o comunidad beneficiada; dicho aval también se anexaba al propio documento del Convenio (SPP, 1989:5). Esto sentó las bases para que la programación se trasladara al nivel de la comunidad, saltando a las autoridades locales tanto estatales como municipales. La ineficiencia de las estructuras administrativas locales, evidentes con las políticas de descentralización delamadrista, fue resuelta pasando por arriba de dichas estructuras. Se estableció así una relación directa entre el Presidente y los beneficiarios del PRO. NASOL; si bien dicha relación ya se había establecido a través del PIDER y COPLAMAR, con Salinas fue llevada al extremo.

La capacidad de moldear y dar cauce institucional a las demandas de grupos de población potencialmente conflictivos del PRONASOL fue todo un éxito desde su primer año de funcionamiento. Al presentar su Primer Informe de Gobierno, el Presidente Salinas reconoció que se habían realizado 44 mil obras a través de Solidaridad, y anunció que el presupuesto asignado al Programa se duplicaría para 1990.

Solidaridad comenzó, entonces, a crecer. Para 1994, el PRONASOL era utilizado para realizar, con la participación directa de los propios beneficiarios, obras de infraestructura básica (pavimentación, agua potable, alcantarillado, electricidad, etc.), así como la rehabilitación y el mantenimiento de planteles educativos, centros de salud comunitarios, hospitales, centros penitenciarios, unidades habitacionales obreras y espacios deportivos. Sirvió también para apoyar pequeños proyectos productivos y proyectos ecoló- 
gicos; y para asignar apoyos económicos y en especie a una serie de grupos de población ubicados como: niños pobres, campesinos de zonas de temporal, jubilados, periodistas, enfermeras, cafeticultores, mujeres e indígenas, entre otros. Además de apoyos para mejoramiento de vivienda y programas de desarrollo regional. Siempre bajo la sombra protectora del titular del Poder Ejecutivo, el Presidente Carlos Salinas.

Para ese momento era claro que el PRONASOL estaba lejos de ser un simple programa, y se convirtió en un mecanismo de asignación discrecional de los recursos económicos y materiales gubernamentales para atender demandas sociales específicas a través de los recursos presupuestales del Ramo XXVI. Demandas que, como el Presidente Salinas de forma constante reiteraba, los sistemas administrativos tradicionales no habían podido resolver satisfactoriamente.

El alzamiento armado en Chiapas, el primero de enero de 1994, puso en evidencia que los pobres del país seguían siendo tan pobres y marginados como al inicio del gobierno de Carlos Salinas. La guerrilla surge en los municipios de más alta marginación, precisamente en el estado donde el Programa asignó mayor cantidad de recursos y donde se crearon, según cifras del PRONASOL, el mayor número de Comités de Solidaridad ${ }^{10}$. EI PRONASOL se convirtió en el centro del debate. Se recrudecieron las críticas que lo acusaban de ser un programa populista, clientelar, electorero. Los enormes esfuerzos del Presidente Salinas por crear una estructura organizativa en torno de los Ilamados Comités de Solidaridad ${ }^{11}$ mostró su enorme fragilidad. El principio del movimiento chiapaneco fue el inicio también de la caída estrepitosa del PRONASOL y del Presidente Salinas, su principal promotor.

Sin embargo, más allá del nivel coyuntural, vale la pena destacar la contribución del Solidaridad al cambio estructural del país. En el anterior sentido podemos mencionar tres hechos importantes: 1) Ha quedado documentado que la labor realizada por el PRONASOL en zonas donde el voto le fue adverso al Partido Revolucionario Institucional, durante las elecciones presidenciales en 1988, le permitió a este partido ganar las elecciones federales de 1991 y recuperar el con-

10 Dichos Comités pueden definirse como unidades grupales organizadas con el fin de aspirar y acceder a los beneficios de las obras y recursos económicos gubernamentales manejados vía PRONASOL. Respecto del número de Comités oficialmente formados en Chiapas, ver: "La organización social en el Programa de Solidaridad" (Gaceta de Solidaridad; 1994).

11 Los esfuerzos por crear una estructura organizativa a través de PRONASOL fueron muy importantes. La evaluación de fin del sexenio presentó el esquema de actuación de Solidaridad como constituido por cinco niveles de organizaciones: el Comité de obra, identificado como el Comité de Solidaridad ordinario; el Comité Comunitario, constituido por un grupo de Comités de obra; las Coordinadoras de Comités, integradas tanto por Comités de obra como por Comités Comunitarios; los Consejos Municipales, considerados instancias superiores constituidas por un conjunto de Coordinadoras, y los Consejos Estatales, integrados por Coordinadoras Regionales y Consejos Municipales (Barajas, 1997). 
trol del Congreso (Molinar y Weldom, 1994). Con tal control, el Presidente Salinas promovió una serie de reformas constitucionales de enorme trascendencia como la reforma del artículo 27 constitucional $^{12}$, entre otros igualmente importantes, 2) EI PRONASOL, visto como una fórmula de gobernabilidad, proporcionó márgenes de acción al gobierno salinista para llevar a cabo una serie de transformaciones: adelgazamiento del Estado (bajo el lema de que "un Estado propietario no es el más justo"), la reducción de las regulaciones del mercado y la firma del Tratado de Libre Comercio con Estados Unidos y Canadá. Los cambios en conjunto lograron replantear la función del Estado y modificar su patrón de rectoría: sustituir el estatismo nacionalista de la Revolución Mexicana por la idea neoliberal de una economía de mercado y un Estado encargado sólo de proporcionar los satisfactores que ningún agente particular puede ofrecer al interés general y 3 ) A partir del PRONASOL, el Presidente Salinas impulsó un nuevo modelo de política social. Planteó que ésta debía de dejar de ser expansiva y vinculada a la política de desarrollo para asumir un carácter selectivo. Uno de los blancos de la crítica del gobierno salinista fueron las políticas de gasto indiferenciado, a las cuales atribuyó los trastornos y debilidad de las fi- nanzas publicas; la pobreza era resultado de los compromisos "equivocados" del Estado posrevolucionario con el bienestar social general. Entonces introdujo las políticas de gasto focalizado, la obligación del Estado, según Salinas, debía ser con los más pobres únicamente.

Esta diferencia, que puede ser considerada de matiz, supuso la ruptura del pacto posrevolucionario que implicaba la obligación del Estado de velar por el bienestar de los trabajadores, campesinos y sectores populares en general. Lo anterior fue posible, porque el PRONASOL cambió las formas de interlocución tradicional, ya no eran los sindicatos o las organizaciones corporativas del Partido Revolucionario Institucional con quienes negociaba el Presidente una base de apoyo social para su proyecto de gobierno sino directamente con los miembros de los Comités de Solidaridad, todos reunidos bajo la categoría de pobres (Barajas, 1997). De esta manera, además, el Programa proporcionó, desde las esferas del poder, una nueva identidad a estos grupos: ya no eran obreros o campesinos sino sencillamente pobres los beneficiarios de los programas sociales (Bascones, 1997). Así, las condiciones para instrumentar programas focalizados estaban establecidas.

12 Dicha reforma estableció el fin del reparto agrario, la apertura del campo a sociedades mercantiles, la libertad de asociación y venta de las tierras de los ejidos y la creación de tribunales agrarios. De esta manera acabó con uno de los sustentos ideológicos del Estado posrevolucionario, donde se reconocía la propiedad social a través del ejido y la facultad presidencial de asignar y expropiar tierras por consideraciones de utilidad pública. 


\section{Las Políticas Neoliberales de Atención a la Pobreza: el PROGRESA}

Esta tercera etapa abarca de 1995 al gobierno actual, e incluye el gobierno de Ernesto Zedillo y el de Vicente Fox. Por primera vez en la historia de México, un programa de atención a la pobreza se mantiene de un sexenio a otro, lo cual refleja las continuidades del modelo neoliberal en su conjunto. Luego de quedar sentadas las bases de tal modelo, en esta etapa se está configurando un patrón de política social propio del mismo, cuyo elemento central es el PROGRESA, hoy OPORTUNIDADES.

\subsection{El Programa de Educación, Salud y Alimentación}

Teniendo como marco la amenaza de un conflicto armado en Chiapas, el inicio del gobierno de Ernesto Zedillo (1994-2000) resultó ser particularmente conflictivo. El país estaba cimbrado por la violencia política tras los asesinatos del candidato presidencial del PRI y del presidente del partido; y recién iniciado el sexenio, una nueva crisis económica en diciembre de $1994^{13}$.

Las mutuas acusaciones respecto de quién había sido el responsable de la nueva crisis económica provocó la ruptura entre el expresidente Salinas y el Pre- sidente Zedillo. Tal ruptura explica, en parte, el que el tema del "combate" a la pobreza haya sido eliminado del discurso gubernamental. Se impuso la tradición presidencialista mexicana de deshacerse de todo aquello que remitía al sexenio anterior. Sin embargo, la mencionada ausencia no fue solo producto de una lucha por el poder, sino representaba también un cambio en la concepción de cómo enfrentar el problema de la pobreza y el lugar que este problema ocupaba dentro de las prioridades del nuevo gobierno.

La administración zedillista reconoció de forma tácita que el problema de la pobreza no se podía resolver, lo que podíamos aspirar es a buscar la "igualdad de oportunidades" atendiendo a los pobres extremos con subsidios perfectamente focalizados y dirigidos a la demanda. Su preocupación más grande: mejorar la relación costo-impacto de los subsidios y evitar que dichos subsidios interfieran en el libre funcionamiento del mercado.

De manera específica, los cambios apuntaron hacia dos direcciones: la descentralización de los recursos presupuestales del Ramo XXVI, "Desarrollo Regional y Solidaridad" y la puesta en marcha de un programa que buscaba bajar los niveles de desnutrición de los grupos de población más vulnerables: niños menores de 5 años y mujeres lactantes de las familias identificadas como las más pobres del país.

13 "Los ingresos de capital se canalizaron principalmente a instrumentos de corto plazo, lo que les conferiría una gran volatilidad. Los conflictos políticos de 1994 y el insuficiente ingreso de capital minaron las reservas de la nación y condujeron otra vez a la devaluación abrupta, al ajuste y a la recesión aguda en 1995" (Valencia y Aguirre, 1998:77). 
Respecto a la descentralización de los recursos del Ramo XXVI. Al inicio de su gobierno, el Presidente Zedillo modificó las atribuciones hasta ese momento asignadas a la Secretaría de Desarrollo Social (SEDESOL). La Secretaría había sido la instancia responsable, desde el momento de su creación en mayo de 1992, de la programación, autorización, entrega, seguimiento y evaluación de los recursos del Ramo XXVI, correspondientes a PRONASOL. Algunos investigadores, llegaron a considerarla una estructura administrativa creada ex profeso por Carlos Salinas para cobijar al PRONASOL (Hernández, 1993).

De acuerdo con la reforma a la Ley Orgánica de la Administración Pública Federal (28 de diciembre de 1994), la SEDESOL se mantuvo como responsable de la atención de los grupos marginados y del mejoramiento de las condiciones de vida de la población; transfiriendo a otras dependencias tareas diversas que no estaban vinculadas con ese objetivo. Por ejemplo, la política inmobiliaria de la administración pública federal y la operación del registro público de la propiedad federal pasaron a la Secretaría de Contraloría y Desarrollo Administrativo; el Instituto Nacional de Ecología, la Procuraduría del Medio Ambiente y la Comisión Nacional para el Uso y Conocimiento de la Biodiversidad se trasladaron a la Secretaría de Medio Ambiente, Recursos Naturales y Pesca. Por último, a la Secre- taría de Educación Pública se le confió el control de sitios y monumentos del patrimonio cultural (Diario Oficial, 28 de diciembre de 1994). Al mismo tiempo, SEDESOL asumió las atribuciones relativas a la industrialización de la leche para ser distribuida a familias de escasos recursos, la comercialización de bienes de consumo popular y del programa de subsidio de la tortilla ${ }^{14}$, que habían sido responsabilidad de la Secretaría de Comercio y Fomento Industrial hasta ese momento.

De conjunto, la reforma confirió a la SEDESOL un carácter fundamentalmente normativo. Dejó de ser la gran intermediaria entre el Presidente de la República y los representantes de las comunidades oficialmente reconocidos (Comités de Solidaridad), para limitarse a establecer y coordinar los cauces y procedimientos de colaboración entre la Federación, los gobiernos estatales y municipales (Rojas, 1994). Asimismo, a partir de la reforma, se planteó la necesidad de transferir a los municipios los recursos y atribuciones del Ramo XXVI, relativas a dotación de infraestructura social básica antes realizadas a través de PRONASOL. Es decir, agua potable, alcantarillado, drenaje y letrinización, electrificación, urbanización, construcción y conservación de infraestructura educativa básica, construcción y conservación de infraestructura de salud, y construcción de caminos e infraestructura rural.

14 Respectivamente, Leche Industrializada CONASUPO, LICONSA; Distribuidora CONASUPO, DICONSA; y Fideicomiso para la Liquidación del Subsidio a la Tortilla, FIDELIST (Diario Oficial, 1994). 
Debemos entender las anteriores transformaciones como parte de un proceso de racionalización del funcionamiento de la administración pública federal, el cual buscaba fomentar el carácter regulador del Estado, y reducir su participación directa en el ámbito del mercado. Por otro lado, también se buscaba reducir los niveles de centralismo y discrecionalidad presidencial impuestos por el PRONASOL. Por ejemplo, para el ejercicio presupuestal de 1995, por primera vez, se utilizó una fórmula matemática basada en indicadores de pobreza de los estados y municipios del país para distribuir los recursos relativos al mencionado Ramo presupuestal. Cabe destacar que no se descentralizaron el total de los recursos sino que fue un proceso gradual, el cual culminó en 1998 con la creación del Ramo del presupuesto 33, llamado Aportaciones Federales para Entidades Federales y Municipios.

La creación del Ramo 33 resultó un avance importante en términos formales. Primero, porque los recursos para infraestructura social básica fueron entregados por Ley a los gobiernos locales de acuerdo a su grado de marginación, eliminando el manejo político al momento de la asignación de tales recursos. Segundo, acabó con la etiquetación de los recursos económicos públicos desde la Presidencia de la República. A partir de ese momento, serían las autoridades municipales, ya no la federación (más específicamente el Presidente), quienes determinaría las obras que habrían de realizarse. El problema fue que los recursos siguieron siendo tan reducidos que las modificaciones en su asignación no tuvie- ron ningún impacto positivo en el bienestar de la población.

El segundo eje de transformación mencionado fue la instrumentación de lo que inicialmente se presentó como un programa nutricional, mismo que buscaría "focalizar en los aproximadamente 14 millones de personas que se encuentran en situación de pobreza extrema, con énfasis en los sectores más vulnerables como son niños menores de 5 años, mujeres embarazadas o en lactancia, indígenas y discapacitados" (Poder Ejecutivo Nacional, 1995:23).

Tal preocupación se insertó dentro de los esfuerzos gubernamentales por eliminar todos los subsidios generalizados. Dicho proceso comenzó desde el primer año de la administración zedillista. Por ejemplo, ese primer año, 1995, el gobierno zedillista convirtió el Programa de Subsidio al Consumo de Tortilla en un subsidio selectivo. Se acabó el que se otorgaba a la cadena producción-consumo de tortilla, para pasar a ser un subsidio a la demanda que no interferiría con el libre funcionamiento del mercado. Los recursos ya no fueron entregados a los productores de maíz sino a las familias que comprobaran tener ingresos menores a dos salarios mínimos, el padrón se integró por 2.8 millones de familias en situación de pobreza.

Dentro del anterior marco surgió el Programa de Educación, Salud y Alimentación (PROGRESA), la puesta en marcha del Programas no fue un proceso sencillo. Primero, porque coexistían dos grupos políticos dentro la administración pública: reformistas sociales (quienes habían instrumentado el PRONASOL), y la 
línea tecnocrática (cercanos al Presidente Zedillo), quienes buscaban imprimir características distintas al programa (Valencia y Aguirre, 1998). La línea tecnocrática al final prevaleció. Segundo, no se contaba con información estadística para concentrar los recursos en una población de beneficiarios claramente identificados. PIDER, COPLAMAR y PRONASOL fueron programas que respondieron a grupos sociales con capacidad de organizarse y demandar, no se trabajaba con padrones de beneficiarios ubicados por debajo de la línea de pobreza.

PROGRESA arrancó en agosto de 1997 y se mantiene hasta hoy con un nuevo nombre: OPORTUNIDADES. Este programa parte de la idea de que el desarrollo comunitario es consecuencia del fortalecimiento de las capacidades de los individuos y las familias. Por ello, se apoya a la familia, ya no a la comunidad como sucedió con programas anteriores. Se sustituyó la oferta de bienes o servicios por parte del gobierno (que en teoría supone una interferencia para el funcionamiento del mercado), por transferencias monetarias familiares, bajo el supuesto de que las personas hacen un uso racional de los recursos; y se estableció una relación "contractual" entre los beneficiarios individuales y el gobierno, asumiendo cada parte ciertos derechos y obligaciones (PROGRESA, 2000).

Tres componentes lo integran: EDUCATIVO, se otorgan becas educativas para cada uno de los hijos e hijas de las familias beneficiarias entre el tercer grado de primaria y el tercero de secundaria; y se da un apoyo en dinero para la adquisición de útiles escolares. SALUD, se proporciona de manera gratuita un paquete básico de salud, y se proporcionan pláticas en materia de salud nutrición e higiene; y el ALIMENTARIO, se otorga un suplemento alimenticio a todos los niños de menos de dos años, para niños desnutridos de entre 3 y 5 años, y para todas las mujeres embarazadas o lactantes, y se asigna una ayuda monetaria a las titulares para complementar los ingresos de la familia. El tope máximo del apoyo económico fue aproximadamente de 855 pesos al mes por familia en el 2000. Tal monto lo alcanzaron las familias que tenían varios hijos en la escuela (el monto de la beca oscilaba entre 95 pesos en tercero de primaria y 350 pesos en tercero de secundaria), el monto promedio de apoyos para el año 2000 ascendió a 3676 pesos al año, es decir, 306 pesos al mes (PROGRESA, 2001:16).

EI PROGRESA trabaja en las localidades rurales marginadas que cuentan con primaria y secundaria y clínicas de salud. Se realiza una encuesta a todas las familias de las comunidades ubicadas estadísticamente como de alta marginación y mediante una fórmula matemática se selecciona a las beneficiarias. Para el año 2002, se planea comenzar a atender zonas urbanas. Por su parte, las familias están obligadas a enviar a sus hijos a la escuela; asistir todos los miembros a las unidades médicas, de acuerdo con una frecuencia de visitas establecidas de antemano; y a renunciar a cualquier otro tipo de subsidio federal que tengan 0 les sea ofrecido.

Considerando todo lo anterior, es claro que el PROGRESA, hoy OPORTUNIDADES, es un programa de atención a 
la pobreza, no de "superación" de la misma. Si analizamos los diferentes componentes del Programa observamos que es un subsidio directo que busca hacer atractiva a las familias en pobreza extrema, la opción de mandar sus hijos a la escuela. No olvidemos que a pesar de que la escuela es gratuita implica un costo en libros, ropa, desayuno, entre otros gastos; $y$ un costo de oportunidad (los niños podrían trabajar junto con sus padres o ser enviados a la ciudad a emplearse), que esas familias no pueden solventar en un número importante de casos.

El gobierno zedillista, y hoy el de Vicente Fox, buscan con PROGRESA-O PORTUNIDADES, contar con mano de obra con mayores niveles educativos y menor deterioro por problemas alimenticios y de salud para que se incorporen al mercado de trabajo y salgan de la pobreza. El problema es que no se ha atendido el problema de la mala calidad de los servicios de salud y educación que reciben los más pobres en México, por otro lado, como anota Julio Boltvinik "No es la educación, como mucha gente cree, la que resuelve la pobreza. La educación ayuda si las condiciones económicas son adecuadas" (Boltvinik, 2002:15). México sufre desde el año 2000, una situación de estancamiento económico que nos lleva a esperar "que si la economía no crece en este sexenio vamos a terminar con 9 millones más de pobres" (Boltvinik, 2002:16). Éstos se sumarían a los 59.07 millones de mexicanos reconocidos como pobres por la actual administración, de una población de 101 millones de personas.

Recientemente, la SEDESOL fijó los parámetros de medición de la pobreza utilizada por el gobierno federal y determinó que 53.7 por ciento de la población es pobre, entre los cuales 24.7 millones de personas son pobres extremos, es decir, no cuentan con ingresos para cubrir sus necesidades alimentarias (La Jornada, 14 de agosto del 2002). Para el 2001, PROGRESA atendió a 15.6 millones de personas, otorgándoles en promedio el 10 por ciento del costo de la canasta alimentaria mínima según los propios cálculos del gobierno, la cual es de 105 pesos diarios por familia. En pocas palabras los esfuerzos gubernamentales son extremadamente limitados dada la dimensión del problema.

Llama la atención que los dos últimos gobiernos han intentado convertir el problema de la pobreza en México en un problema "técnico", de definición de líneas de pobreza e instrumentación de políticas cada vez más focalizadas. Lo anterior forma parte de los esfuerzos por darle credibilidad a los gobiernos neoliberal al hacer más "transparente" y "eficiente" la asignación y ejercicio del gasto social. Al cubrir a los programas sociales con un aló de "neutralidad política" y de "precisión técnica" buscan imponer una nueva forma de legitimidad estatal. El problema es que treinta años de experiencias similares muestran que los programas de atención de la pobreza no son alternativas reales para sacar a las personas de su situación de marginación y atraso, es necesario establecer una clara vinculación entre política económica y política social, indispensable para elaborar una estrategia verdadera de combate a la pobreza, pero ese debate no se plantea. 


\section{Conclusiones}

Resulta muy difícil dada la extensión del periodo analizado y el carácter de primer acercamiento de la investigación, el plantear conclusiones definitivas. Sin embargo, consideramos importante anotar una serie de ideas que se desprenden del trabajo. En particular, referirnos a la complejidad de los procesos de transformación del aparato estatal y a la existencia de inercias institucionales. Cuando destacamos que las políticas de atención a grupos de población específicos en situación de pobreza no surgen con el modelo neoliberal sino una década antes, vemos como una recomendación de los organismos financieros internacionales se adapta a las necesidades y condiciones políticas, económicas y administrativas particulares de nuestro país. Se dan políticas selectivas pero de corte populista, las cuales funcionaron a partir de las demandas de los grupos de población pobres, siempre organizados por las dependencias de la administración pública federal en comités de beneficiarios. Conforme se ahondaba la crisis de los mecanismos corporativos tradicionales (los sectores campesino, obrero y popular del Partido Revolucionario Institucional), se vuelven más importantes tales programas. Utilizados con el fin de reactivar el modelo posrevolucionario de desarrollo, posteriormente serían utilizados, con el PRO. NASOL, en el sexenio de Carlos Salinas, para sentar la bases de modelo neoliberal. Lo anterior no resulta del todo sorprendente, recordemos que las políticas gubernamentales se desarrollan dentro de marcos normativos, jurídicos e institu- cionales propios de la administración pública, cuyas transformaciones responden a la lógica de la misma; un ejemplo claro es la evolución de los recursos presupuestales del Ramo XXVI. La creación del PROGRESA, en 1997, se da luego de quince años de iniciados los cambios de modelo, como anotamos, las condiciones políticas, ideológicas y administrativas estaban dadas para que apareciera un programa propio del modelo neoliberal.

Para finalizar, nos interesa anotar que el presente ejercicio resultó para nosotros muy productivo en dos sentidos: en términos académicos, porque fue un acercamiento al funcionamiento de la administración pública y una reconstrucción histórica de las políticas de atención a la pobreza ubicadas dentro de un plano general que son los modelo de desarrollo. Pero también lo fue en términos políticos, porque nos muestra que luego de treinta años de experiencias fallidas en materia de atención de la pobreza, resulta claro que el debate en torno al "combate", "ataque" o "superación" de la pobreza no debemos plantearlo centrado en el funcionamiento de este tipo de programas sino en un marco más amplio referido a los efectos del modelo de desarrollo prevaleciente, en este caso el neoliberal, y a la necesidad de transformarlo.

\section{Bibliografía Citada}

Aguilar Villanueva, Luis (1996), "El federalismo mexicano: funcionamiento y tareas pendientes" en Revista Mexicana de Sociología. Año LVIII, Núm. 3. México.

Arteaga, Javier (1985), "El Sistema Alimentario Mexicano: una perspectiva política", en Estudios Sociológicos de EI 
Colegio de México, Vol. III, Núm. 8. México.

Barajas, Gabriela (1997), "Comités de Solidaridad en México. Surgimiento y evolución de la estructura organizativa promovida a partir del Pronasol", en Estudios Sociológicos de El Colegio de México. Vol. XV, Núm 45. México.

Bascones, Luis Miguel (1997), "La exclusión participativa. Pobreza, gobierno y saber en el Programa Nacional de Solidaridad". Universidad Complutense. Madrid. Mimeo.

Boltvinik, Julio (1999), Periódico La Jornada. 21 de junio. México.

Boltvinik, Julio (2002), Periódico La Jornada. 15 de agosto. México.

Campos, Julieta (1995), ¿Qué hacemos con los pobres? Aguilar. México.

Carrasco, Rosalba y Enrique Provencio (1988), "La política social, 1983-1988 y sus principales consecuencias". Investigación Económica, abril-junio. México.

Castell Cancino, Jorge y Fernando Rello Espinosa (1981), "Las desventuras de un proyecto agrario: 1970-1976", en: Rolando Cordera (coord) Desarrollo y crisis de la economía mexicana. FCE, México.

Ciesas-Progresa (1998), Alivio a la pobreza. Análisis del Programa de Educación, Salud y alimentación dentro de la política social. Ciesas. México.

Comisión Nacional de Subsistencia Populares (CONASUPO) (1982), Programa CONASUPO-COPLAMAR de Abasto a Grupos Marginados. Manual del Supervisor. CONASUPO. México.

Consejo Consultivo del Programa Nacional de Solidaridad (1991), El combate a la pobreza. El Nacional. México.

COPLAMAR (1977A), Bases para la Acción 1977-1982 de la Coordinación General del Plan Nacional de Zonas
Deprimidas y Grupos Marginados. COPLAMAR. México.

COPLAMAR (1977B), COPLAMAR. COPLAMAR. México.

Diario Oficial (1994), 28 de diciembre. México.

Dresser, Denise (1997), "En busca de la legitimidad perdida. Pronasol, pobreza y política en el gobierno de Salinas", en: Gabriel Martínez (comp.) Pobreza y política social en México. ITAMFCE. México.

Gaceta de Solidaridad (1994), Órgano de Información del Programa Nacional de Solidaridad, año $\mathrm{V}$, núm. 111, 15 de noviembre. México.

Gutiérrez Garza, Estela (coord)(1989), La crisis del Estado de Bienestar. UNAM-Siglo XXI. México.

Hernández, Rogelio (1993), "La administración al servicio de la política: la Secretaría de Programación y Presupuesto", en: Foro Internacional, núm. 1, enero-marzo. México.

IMSS-COPLAMAR (s/f), Situación del estado de salud del grupo de población materno infantil amparada por el Programa IMSS-COPPLAMAR. México, mimeo.

INI (1977), Informe de labores 1976-1982. INI. México.

Instituto Nacional Indigenista, INI (1982), Informe de labores 1976-1982. INI. México.

Medina Peña, Luis (1994), Hacia el nuevo Estado. México, 1920-1994. FCE. México.

Molinar Horcasitas, Juan y Jeffrey A Weldom (1994), "Electoral Determinants and Consequences of National Solidarity" en: Wayne Cornelius and Jonathan Fox. Tranforming State-Society Relations in Mexico. USA, Universidad de California.

Montes de Oca Luján, Rosa Elena (1981), "La cuestión agraria y el movimiento cam- 
pesino: 1970-1976", en: Rolando Cordera (coord) Desarrollo y crisis de la economía mexicana. FCE. México.

Ortega Lomelin, Roberto (1988), El nuevo federalismo. La descentralización. Edit. Porrúa. México.

Peniche, Antonio (1992), "El Pronasol: algunas notas y reflexiones generales", en EI Cotidiano, Núm 49, julio-agosto. México.

Poder Ejecutivo Nacional (1995), Plan Nacional de Desarrollo (1995-2000). México.

PROGRESA (2000), Reglas de Operación del Programa de Educación, Salud y Alimentación. Sedesol.

PROGRESA (2001), Programa de Educación, Salud y Alimentación. Mimeo.

Rojas Gutiérrez, Carlos (1994), "Secretaría de Desarrollo Social". Revistas de Administración Pública. México.

SEDESOL (1993), Cronología del inicio de los principales programas y actos del Programa de Solidaridad, de diciembre de 1988 a mayo de 1993. México. Mimeo.
SPP (1985), Antología de la Planeación en México. SPP. México.

SPP (1989), Informe a los miembros del Consejo Consultivo del PNS. México. Mimeo.

Szekely, Miguel (1993), El Programa Nacional de Solidaridad en México. Programa Mundial del Empleo. Núm. 384. México.

Trejo, Guillermo y Claudio Jones (1993), Contra la pobreza. Cal y Arena. México.

Valencia Lomelí, Enrique y Rodolfo Aguirre Riveles (1998), "Discursos, acciones y controversias de la política gubernamental frente a la pobreza", en Rigoberto Gallardo y Joaquín Osorio Los rostros de la pobreza. El debate. ITESO-UIA. México.

Vázquez Alanís, Fernando (1989), "Las esferas de gobierno y sus mecanismos de coordinación", en: Jose Chanes Nieto (et alt). Ensayos sobre la modernidad nacional. Diana. México.

Woldenberg, José y Mario Huacuja (1986), "EI sexenio de Luis Echeverría”, en: Evolución del Estado mexicano. Tomo III. El caballito, México. 